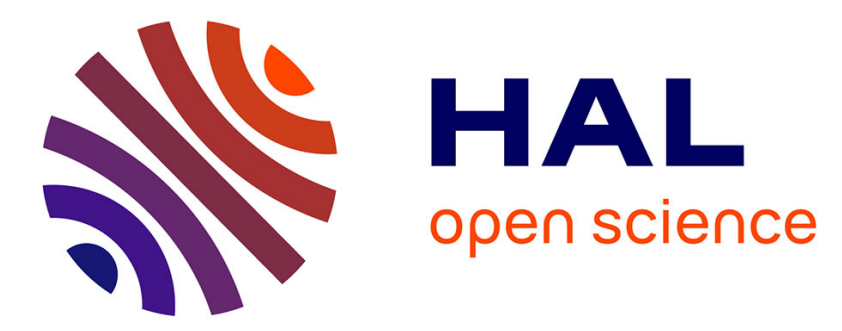

\title{
Kinetic study of the linear ising chain. comparison with ultrasonic absorption measurements in solutions of polypeptides
}

\author{
Y. Dormoy, R. Cerf
}

\section{- To cite this version:}

Y. Dormoy, R. Cerf. Kinetic study of the linear ising chain. comparison with ultrasonic absorption measurements in solutions of polypeptides. Journal de Physique Lettres, 1976, 37 (10), pp.265-270. 10.1051/jphyslet:019760037010026500 . jpa-00231289

HAL Id: jpa-00231289

https://hal.science/jpa-00231289

Submitted on 1 Jan 1976

HAL is a multi-disciplinary open access archive for the deposit and dissemination of scientific research documents, whether they are published or not. The documents may come from teaching and research institutions in France or abroad, or from public or private research centers.
L'archive ouverte pluridisciplinaire $\mathbf{H A L}$, est destinée au dépôt et à la diffusion de documents scientifiques de niveau recherche, publiés ou non, émanant des établissements d'enseignement et de recherche français ou étrangers, des laboratoires publics ou privés. 


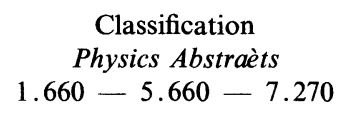

\title{
KINETIC STUDY OF THE LINEAR ISING CHAIN. COMPARISON WITH ULTRASONIC ABSORPTION MEASUREMENTS IN SOLUTIONS OF POLYPEPTIDES
}

\author{
Y. DORMOY and R. CERF \\ Laboratoire d'Acoustique Moléculaire $\left(^{*}\right)$ Université Louis-Pasteur, \\ 4, rue Blaise-Pascal, 67070 Strasbourg Cedex, France \\ (Reçu le 6 février 1976, révisé le 28 juin 1976, accepté le 8 juillet 1976)
}

\begin{abstract}
Résumé. - La cinétique de la chaîne linéaire d'Ising a été traitée sans autre approximation que la fermeture aux triplets et la réduction à quatre des équations cinétiques. On a étudié plus particulièrement l'influence des fluctuations du nombre de séquences ininterrompues d'éléments identiques en introduisant une variation de volume molaire $\overline{\Delta V}$ liée à ces fluctuations. Par un choix adéquat des paramètres cinétiques, il est possible de prendre en compte au moins partiellement des interactions à longue distance et des effets de bouts de chaîne. La théorie est appliquée à l'étude cinétique de la transition hélice-pelote des polypeptides. Pour une chaîne relativement courte d'acide poly-1-glutamique de masse moléculaire $M=12000$, qui est encore longue devant la distance de coopérativité, le déplacement du pic d'absorption ultrasonore dû à la relaxation 1 vers les hélicités élevées va de pair avec l'apparition d'une contribution non négligeable de l'absorption due à une seconde relaxation. Les résultats de la théorie sont en accord avec les mésures existantes, et l'on a pu évaluer le volume $\overline{\Delta V}$.
\end{abstract}

\begin{abstract}
A study of the kinetics of the linear Ising chain has been carried out using as sole approximations the triplet closure and four kinetic equations. In particular, we have examined the effect of fluctuations of the number of uninterrupted sequences of the like elements by introducing a molar volume change $\overline{\Delta V}$ associated with these fluctuations. Long range interactions and end effects can be partially accounted for by a suitable choice of the kinetic parameters. The theory is applied to the kinetic study of the helix-coil transition of polypeptides. For a relatively short poly-1-glutamic acid chain of molecular weight $M=12000$ (which is still long compared with the cooperativity length) the shift of the ultrasonic absorption peak due to relaxation 1 is accompanied by a nonnegligible contribution of the absorption associated with a second relaxation. The theoretical results agree with existing measurements, and we have evaluated the volume $\overline{\Delta V}$.
\end{abstract}

1. Introduction : time-dependent statistics of the linear Ising chain. - It is well known that various biological functions of nucleic acids and proteins are governed by conformations which are extremely sensitive to small external effects. Such high sensitivity is the result of the cooperative nature of the elementary steps of conformational changes.

The importance of the kinetic aspects of conformational changes is equally well known, e.g., in the transfer of genetic information or in the process of enzymatic reactions. Thus, it is interesting to carry out kinetic studies based on simple models that exhibit the cooperative aspects of the problem.

Linear systems are commonly found among biopolymers and they furnish good examples that readily lend themselves to both theoretical and expe-

(*) E.R.A. au C.N.R.S. rimental studies. Efforts have therefore been directed by many authors towards an understanding of the time-dependent statistics of the simplest cooperative linear chain in which the interactions occur between next-neighbors, i.e., the one dimensional Ising lattice. We shall denote by $A$ and $B$ the two possible states of each element of the chain. An example of an $A \rightleftharpoons B$ conformational change will be discussed in section 3 .

In Schwarz's model [1] propagation steps $(\mathrm{AAB} \rightleftharpoons \mathrm{ABB})$ and nucleation steps $(\mathrm{AAA} \rightleftharpoons \mathrm{ABA}$; $\mathrm{BBB} \rightleftharpoons \mathrm{BAB})$ are considered and a molar volume change $\delta V$ is associated with the $\mathrm{A} \rightleftharpoons \mathrm{B}$ conformational change. Consequently, fluctuations of the fraction $p(\mathrm{~B})$ of elements in the B-form produce an overall volume change and hence loss of energy in an ultrasonic wave. In aqueous solutions, the corresponding attenuation coefficient is proportional to [1] :

$$
(\delta V)^{2}[\partial p(\mathrm{~B}) / \partial \operatorname{Ln} s]
$$


where $s$ is the equilibrium constant associated with the $\mathrm{A} \rightleftharpoons \mathrm{B}$ change. For a long, highly cooperative chain, expression (1) is maximum for $s=1$, i.e., for $p(\mathrm{~B})=0.5$.

In the present context we shall use a more general scheme, proposed by one of us [2,3], and in which fluctuations of the number $v$ of uninterrupted A or B-sequences per molecule are also taken into account. This is done by introducing an average molar volume change $\overline{\Delta V}$, which is associated with the loss of one uninterrupted A-sequence per chain.

It can be shown by simple reasoning [2] that the fluctuations of $v$ contribute to the energy loss in an ultrasonic wave and that for relatively large values of $p(\mathrm{~B})$ the corresponding attenuation is proportional to :

$$
-N^{-1} \delta V \overline{\Delta V}(\partial v / \partial \operatorname{Ln} s)
$$

where $N$ is the number of elements (A or B) per chain.

The kinetic theory of the model considered here can be developed quantitatively if the triplet closure approximation recently proposed by Schwarz $[4,5]$ is used. Within this approximation, the probability of any sequence of elements of the chain is expressed in terms of probabilities of doublets and triplets [4]. When the chain is very long, the triplet closure results in the reduction of the number of rate equations, which is a priori of the order of $2^{N}$, to only 4, i.e., those equations which govern the probabilities of singlets, doublets and triplets [4]. The kinetics is then described in terms of four relaxation times and of the corresponding amplitude factors.

For the model that includes the volume change $\overline{\Delta V}$ the triplet closure has been shown [3] to lead to explicit expressions both of the relaxation amplitude $A_{1}$ associated with Schwarz's longest relaxation time $\tau_{1}$ and of the $(\overline{\Delta V})^{2}$-proportional contribution to the amplitude $A_{2}$ associated with the second relaxation time $\tau_{2}[6]$.

Additional information can be obtained by numerical calculations. This will be shown in section 2 to furnish the contributions to $A_{2}$ that are proportional to $(\delta V)^{2}$ and to $\delta V \cdot \overline{\Delta V}$. It also permits a variation in the values of the three kinetic parameters of the model. This possibility will be exploited in section 3 when the model is applied to a concrete example.

2. Results of numerical calculations. - It is recalled that according to Schwarz [1] three kinetic parameters are considered : $k_{\mathrm{D}}$, associated with the propagation step $\mathrm{ABB} \rightarrow \mathrm{AAB} ; \sigma f_{\mathrm{A}} k_{\mathrm{F}}$, associated with the nucleation step AAA $\rightarrow \mathrm{ABA}$ and $\sigma f_{\mathrm{B}} k_{\mathrm{D}}$ associated with the nucleation step $\mathrm{BBB} \rightarrow \mathrm{BAB} ; \sigma$ is the equilibrium nucleation parameter of $\mathrm{Zimm}$ and Bragg [7] and $k_{\mathrm{F}}=s k_{\mathrm{D}}$. Except in figures 5 and 6, the kinetic parameters $f_{\mathrm{A}}$ and $f_{\mathrm{B}}$ are chosen to be equal to 1 .
The non-negligible contributions to the ultrasonic absorption are produced by the relaxations associated with the two longest times $\tau_{1}$ and $\tau_{2}$. Figure 1 shows the contributions $A_{1}$ and $A_{2}$ of relaxations 1 and 2 to the quantity :

$$
\frac{\alpha \lambda}{\pi} \frac{R T}{\rho v^{2} c(\delta V)^{2}}
$$

for a constant frequency such that $\omega \tau_{1 \mathrm{M}}=2.5$; $\omega$ is the circular frequency and $\tau_{1 M}$ the maximum value reached by $\tau_{1}$ when $p(\mathrm{~B})$ is varied.

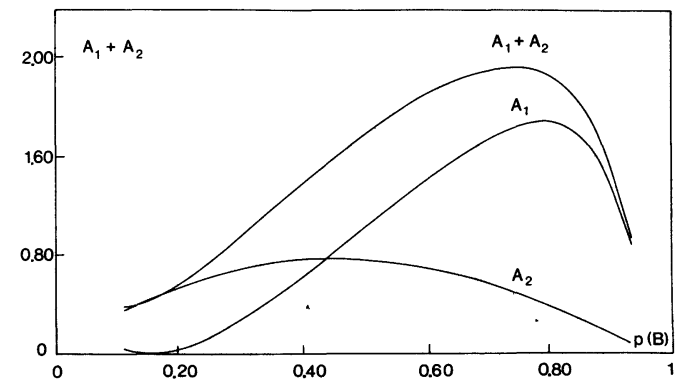

FIG. 1. - Ultrasonic absorption per wavelenght $A_{1}$ and $A_{2}$ for the two relaxation processes 1 and 2, and sum $A_{1}+A_{2}$ plotted against $p$ (B) for $\sigma=5 \times 10^{-3}, \quad N=100, \overline{\Delta V} / \delta V=15, \omega \tau_{1 \mathrm{M}}=2.5$, $f_{\mathbf{A}}=f_{\mathbf{B}}=1$.

In expression (3), $\lambda$ is the wavelength of the ultrasound, $v$ its propagation velocity, $R$ the gas constant, $T$ the absolute temperature, $\rho$ the density of the medium and $c$ the concentration of chain elements in moles per unit volume. The quantities $A_{1}, A_{2}$ and $A_{1}+A_{2}$ are plotted against $p(\mathrm{~B})$ for the above value of $\omega \tau_{1 \mathrm{M}}$ and for $\sigma=5 \times 10^{-3}, N=100$, $\overline{\Delta V} / \delta V=15$. The figure shows the displacement of the absorption peak towards values of $p(\mathrm{~B})$ larger than 0.5 , as already discussed in references [2] and [3]. It is seen that the displacement is due to relaxation 1.

Figure 2 illustrates and compares three different approximations of $B_{1}=A_{1}\left[\omega \tau_{1} /\left(1+\omega^{2} \tau_{1}^{2}\right)\right]^{-1}$, as deduced from the expression for the amplitude factor $A_{1}^{\prime}=-(\delta V)^{2} B_{1} /(R T)$ of relaxation 1 , given

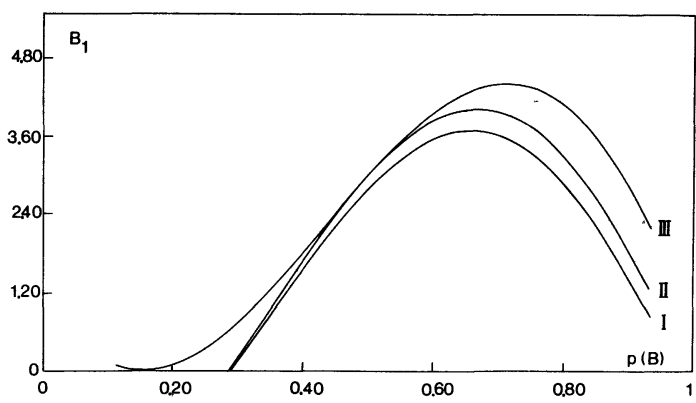

FIG. 2. - Value of $B_{1}=A_{1}\left|\omega \tau_{1} /\left(1+\omega^{2} \tau_{1}^{2}\right)\right|^{-1}$ as a function of $p(\mathrm{~B})$ for the values of the parameters as in figure 1 : Curve $\mathrm{I}$ : approximate value of $B_{1}$ as given by expression (4); Curve II : sum of the $(\overline{\Delta V} / \delta V)^{\circ}$ and $(\overline{\Delta V / \delta V)}$, proportional terms, calculated numerically ; Curve III : exact value of $B_{1}$. 
in reference [3]. At relatively large values of $p(\mathrm{~B})$ we have, to a first approximation [3] :

$$
B_{1}=\frac{\partial p(\mathrm{~B})}{\partial \operatorname{Ln} s}-\frac{2}{N} \frac{\overline{\Delta V}}{\delta V} \frac{\partial v}{\partial \operatorname{Ln} s} ;
$$

i.e., $B_{1}$ is the sum of two terms that are proportional to (1) and (2), respectively. The quantity (4) is represented in figure 2 (curve I) as a function of $p(\mathrm{~B})$ for the preceding values of $\sigma, N$ and $\overline{\Delta V} / \delta V$. Curve II represents $B_{1}$ when the exact values of the terms proportional to $(\overline{\Delta V} / \delta V)^{0}$ and to $(\Delta V / \delta V)$ are retained, and Curve III is calculated using the complete expression of $B_{1}$ mentioned above, i.e., in which the term proportional to $(\overline{\Delta V} / \delta V)^{2}$ is also retained. It is seen that the $(\overline{\Delta V} / \delta V)^{2}$-term enhances the displacement of the absorption peak towards high values of $p(\mathrm{~B})$. For $\overline{\Delta V} / \delta V<10$ expression (4) is an acceptable approximation for $B_{1}$.

Figure 3 represents the value $p_{\mathrm{m}}(\mathrm{B})$ of $p(\mathrm{~B})$ for which $A_{1}$ is largest at a given value of the frequency $F$, plotted against $\overline{\Delta V} / \delta V$ for various values of $\omega \tau_{1 \mathrm{M}}$. The parameters have the same values as before. The dotted lines represent the results of a first approximation published earlier [3].

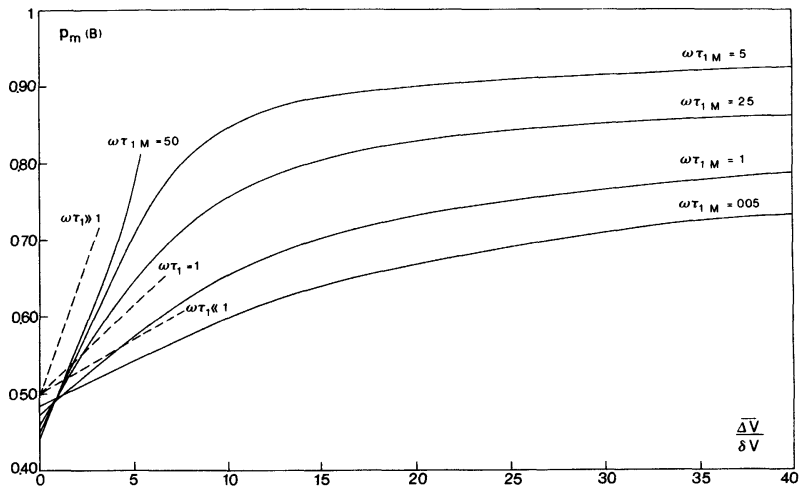

Fig. 3. - Value of $p_{\mathrm{m}}$ (B) (at which $A_{1}$ is a maximum) plotted against $\overline{\Delta V} / \delta V$ for different values of $\omega \tau_{1 \mathrm{M}}$. The parameters have the same values as in figures 1 and 2 . Dotted straight lines represent first approximation results (see reference [3]).

Figure 4 represents $k_{\mathrm{D}}\left(A_{1}+A_{2}\right) / \omega$ which is proportional to $\alpha / F^{2}$ plotted as a function of $p(\mathrm{~B})$ for various values of $\omega \tau_{1 \mathrm{M}}$ (i.e., for various frequencies) and for the values of the parameters already used in the previous figures.

3. Kinetics of the helix-coil transition of poly-Lglutamic acid. - The helix-coil transition of poly- $\alpha$ aminoacids composed of like units (i.e., polypeptides in which each amino acid residue

$$
-\mathrm{CO}-\mathrm{CHR}-\mathrm{NH}-
$$

bears the same side groupe $\mathrm{R}$ ) has been thoroughly investigated with regard to both statistical and kinetic aspects. When dissolved in an appropriate solvent, a helical polypeptide can undergo a sharp transition

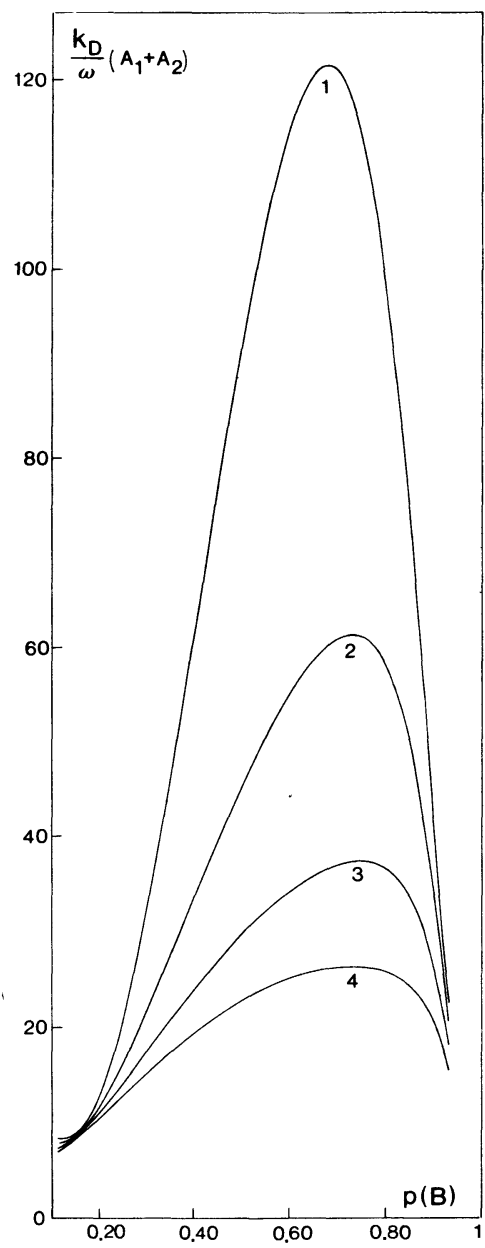

FIG. 4. - A quantity proportional to the amplitude of $\alpha / F^{2}$ due to relaxations 1 and 2 is plotted against $p(\mathrm{~B})$ for $\omega \tau_{1 \mathrm{M}}=1,2,3,4$. The parameters have the same values as in the preceding figures.

to a randomly coiled chain when, for example, the $\mathrm{pH}$ is varied through a certain range.

In the $\alpha$-helical conformation, the peptide nitrogen atom is hydrogen-bonded to the peptide (carbonyl) oxygen atom of the fourth residue down the chain. The randomly coiled conformation results if the hydrogen bonds are severed, whereupon intramolecular rotation occurs.

Each residue of the chain is therefore considered to present essentially two conformations : the B or helical conformation, as exhibited in the $\alpha$-helix, and the $\mathrm{A}$ or coiled conformation, which corresponds to a collection of rotational isomeric forms ; $p(\mathrm{~B})$ now represents the helicity of the chain, which we shall call $\theta$, as usual. With the preceding simplified picture the equilibrium properties of the system can be studied by representing the polypeptide chain as a linear Ising lattice [7].

Schwarz's approach to the kinetic problem amounts to assuming :

1) that we need only consider the fluctuations of helicity;

2) that interactions other than those between adjacent neighbors can be neglected in the kinetics 
if 1) is true, in which case the Ising model can be used for treating the kinetics.

Various experimental results, as reviewed by Schwarz and Engel [8], are in favor of the correctness of 1) for long chains.

A deviation from the behavior described by Schwarz has, however, been observed for a poly-Lglutamic acid of molecular weight $M=12000[9,10]$, thereafter called PLGA 2. For this substance, the molecules contain about 94 monomer units and are still long compared with the cooperativity length $n=\sigma^{-1 / 2}$. We recall that $n$ is the mean number of monomer units in one uninterrupted sequence of like elements at mid-transition. If the currently admitted value of $\sigma$ for PLGA is used, $n$ is of the order of 14 .

The above mentionned experimentally observed deviation from ideal behavior consists in a displacement of the absorption peak from its value $\theta=0.5$ for a long chain (see section 1) to a value $\theta_{m}$ lying between 0.7 and 0.85 .

It has been shown in references [2] and [3] (and can also be seen in figures 1 to 4 ) that the fluctuations of the number $v$ of uninterrupted sequences of like elements per molecule offer a possible explanation of this displacement of the absorption peak. The origin of the mean volume change $\overline{\Delta V}$ that permits one to account for the fluctuations of $v$ is discussed in detail in reference [3]. It was suggested that $\overline{\Delta V}$ results from a redistribution of the segments that were a part of a lost disordered sequence among the other disordered sequences and that forces responsible for the stability and conformational transitions of proteins are involved.

From this scheme it follows that $\overline{\Delta V}$ should increase when the molecular weight decreases. For PLGA 2 a value of $\overline{\Delta V} / \delta V$ of several units has been proposed as a reasonable estimate [3].

It must now be emphasized that if we reject Schwarz's assumption 1), we should, in principle, also reject assumption 2), i.e., it is questionable whether the Ising model is applicable to our problem.

There are observations suggesting that 2) may not be entirely correct, even in situations in which the fluctuations of helicity are the primary factor, i.e., situations in which assumption 1) is correct. We refer in particular to the fact that the numerical value of $k_{\mathrm{D}}$ (or $k_{\mathrm{F}}$ ) as expected by Schwarz (i.e., $10^{10}$ $10^{11} \mathrm{~s}^{-1}$ ) is widely different from those found experimentally by Barksdale and Stuehr for PLGA [11] (i.e., $8 \times 10^{7} \mathrm{~s}^{-1}$ ) and by ourselves (see below). One is thus led to consider $k_{\mathrm{D}}$ as an effective kinetic parameter and this may reflect in particular the fact that the elementary growth process involves rather long-range movements of the chain relative to the solvent.

It should be noted here that, according to Eggers and Funck [12], at concentrations as low as those used by Barksdale and Stuehr (i.e., a few $10^{-3}$ mole of residue/1), and contrary to the result of the latter authors, no maximum of the ultrasonic absorption is observable when $\theta$ is varied. It may be that isolated PLGA molecules collapse to a compact cluster at relatively high values of the helicity, thus producing departures from the behavior described by Schwarz.

On the other hand, such a collapse may be impossible at medium concentrations such as those used by Michels [9, 6] (i.e., of the order of $10^{-1}$ mole residue/1), at which the PLGA molecules cannot be considered isolated and present entanglements. The elementary steps of the Schwarz scheme may in this case remain of primary importance. From this point of view, the necessity of using an effective value of $k_{\mathrm{D}}$ when employing the Ising model may reflect partly intermolecular and long range intramolecular forces.

Long range interactions are of course critical when fluctuations of the number of uninterrupted sequences of like elements are considered, as is the case here. Indeed, in order that two helical sequences may link together they must be properly positioned in space, and this is very improbable [13]. We can, however, take this circumstance into account by choosing an $f_{\mathbf{B}^{-}}$-value that is significantly smaller than unity. One might argue that such a choice of $f_{\mathrm{B}}$ unduly diminishes the probability of the coil nucleation, which does not depend on the above geometrical condition for coalescence of two neighboring helical sequences.

This objection is of little practical consequence, since we shall be compelled to choose $f_{\mathrm{A}}$ larger than unity when the theory is confronted with the measurements. Thus, coil nucleation, for which the probability is crudely approximated in our description turns out to be the less probable process. In any event, it is less probable than the reverse process by a factor $s \sigma^{-1}$ and is even less probable than helix nucleation, as a consequence of the choice of $f_{\mathrm{A}}$. Thus, the important processes in producing fluctuations of $v$ for PLGA 2 are the helix nucleation and its reverse, i.e., the disappearance of a helix nucleus.

It is possible that our scheme reflects the fact that conformational changes are easier at an end of the molecule. However, we have no way of assessing whether $f_{\mathrm{A}}$ should be smaller at high molecular weight since, in the present framework, at high $M$ values only the fluctuations of helicity are of importance. Thus, the values of $f_{\mathrm{A}}$ and $f_{\mathrm{B}}$ are reflected neither in the relaxation time (time $\tau_{1}$ ) nor in the corresponding amplitude. Therefore, when considering the results obtained for a sample of higher molecular weight. (PLGA 1; $M=90000$ ), we shall use the same values of $f_{\mathrm{A}}$ and $f_{\mathrm{B}}$ as for PLGA 2.

In addition to the use of the Ising model itself and to the choice of the $f_{\mathrm{A}}$ and $f_{\mathrm{B}}$-values, the assumptions are those of reference [3], i.e. :

1) when the chain length is not less than the cooperativity length multiplied by a factor of units, the 
four rate equations that govern the relevant probabilities for a long chain can be applied.

2) for smaller chain-lengths, however, we must choose correspondingly larger values of $\overline{\Delta V} / \delta V$, in accordance with the mechanism that we assume is producing $\overline{\Delta V}$.

It will now be shown that with these assumptions we can understand the results obtained for both PLGA 1 and 2, including the hitherto unnoticed fact that the displacement of the absorption peak of relaxation 1 in the $\theta$-scale (or the pH-scale) is concomitant with the appearance of a second relaxation.

Figure 5 is a double logarithmic representation of $\left(A_{1}+A_{2}\right)_{\mathrm{m}}$ (i.e., the maximum of $A_{1}+A_{2}$ when $\theta$ is varied) plotted against $\omega \tau_{1 \mathrm{M}}$. Curve a corresponds to the values of $\sigma, N, \overline{\Delta V} / \delta V, f_{\mathrm{A}}$ and $f_{\mathrm{B}}$ already used. In comparison, curbe $\mathrm{b}$ is obtained for $\sigma=3 \times 10^{-3}, \overline{\Delta V} / \delta V=10$ and $f_{\mathrm{A}}=5, f_{\mathrm{B}}=0.2$. Relaxation 2 is displaced to higher frequencies when $f_{\mathrm{A}}$ is greater than unity. Curve c shows the effect of choosing $f_{\mathrm{B}}=5$ instead of 0.2 . The comparison with the latest measurements of Michels for PLGA 1 and 2 [6] is given in figure 6 . Since only relaxation 1 contributes to the ultrasonic absorption for PLGA 1 and since both samples show comparable absorption at the lower frequencies, relaxation 2 must contribute only slightly in this range for PLGA 2 . Thus $\overline{\Delta V} / \delta V$ must be lower than a limit of the order of 10 . Curve $d$ is similar to $\mathrm{b}$ of figure 5 , except that $f_{\mathrm{B}}$ has been chosen equal to 0.5 . In figure 6 , we have also represented the theoretical curve e for a long chain, using the same values of the parameters as for curve $\mathrm{d}$, except that $N=500$ and $\overline{\Delta V}=0$. The maximum value $\tau_{1 \mathrm{M}}$ of the longest time for the two samples lie so close together that $\omega \tau_{1 \mathrm{M}}$ represents a suitable variable.

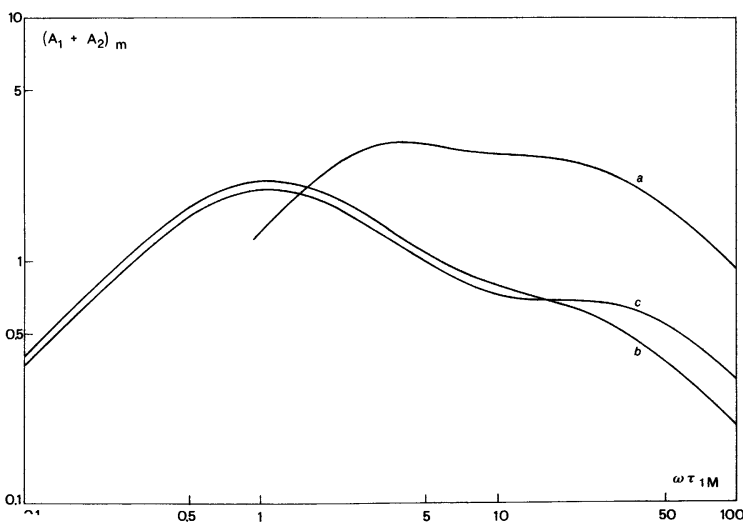

FIG. 5. - The maximum values of $A_{1}+A_{2}$ (reached at variable $\theta$ ) are plotted as a function of $\omega \tau_{1 \mathrm{M}}$ : Curve a : $\sigma=5 \times 10^{-3}$, $N=100, \overline{\Delta V} / \delta V=15, f_{\mathrm{A}}=f_{\mathrm{B}}=1$; Curve b : $\sigma=3 \times 10^{-3}$, $N=100, \overline{\Delta V} / \delta V=10, f_{\mathrm{A}}=5, f_{\mathrm{B}}=0.2 ;$ Curve c $: \sigma=3 \times 10^{-3}$, $N=100, \Delta V / \delta V=10, f_{\mathbf{A}}=f_{\mathbf{B}}=5$

It is noteworthy that the presently available data are in agreement with theory when the same values of $\delta V$ and $k_{\mathrm{D}}$ are chosen for the two samples :

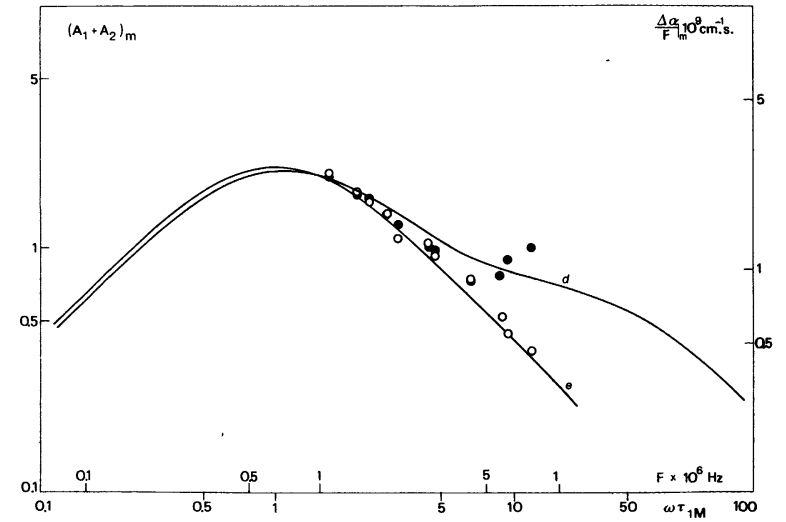

FIG. 6. - Comparison of the theory with the experimental result of Michels for PLGA $1(0 ; N \sim 700)$ and PLGA $2(\bullet ; N \sim 94)$ The maximum conformational contribution to the ultrasonic absorption divided by the frequency, $\left.\frac{\Delta \alpha}{F}\right|_{m}$, is plotted as a function of the frequency for solutions at $2 \times 10^{-2} \mathrm{~g} / \mathrm{cc}$ in $0.2 \mathrm{M} \mathrm{NaCl}$. The theoretical curves are represented as in figure $5:$ Curve $\mathrm{d}$ : $\sigma=3 \times 10^{-3}, N=100, \overline{\Delta V} / \delta V=10, f_{\mathrm{A}}=5, f_{\mathrm{B}}=0.5 ;$ Curve e $\sigma=3 \times 10^{-3}, N=500, \overline{\Delta V} / \delta V=0, f_{\mathrm{A}}=5, f_{\mathrm{B}}=0.5$.

$0.58 \mathrm{cc} /$ mole of monomer $<\delta V<0.7 \mathrm{cc} / \mathrm{mole}$ of monomer

$$
1.7 \times 10^{8} \mathrm{~s}^{-1}<k_{\mathrm{D}}<4.3 \times 10^{8} \mathrm{~s}^{-1} .
$$

Moreover, this value of $\delta V$ is close to those which have been obtained by other methods $[14,15]$ and the $k_{\mathrm{D}}$-values is not very different from that deduced by Barksdale and Stuehr [11] from their ultrasonic measurements.

The results discussed here furnish for the first time an example which suggests an effective contribution of relaxation 2 of the linear Ising chain to the ultrasonic absorption. Furthermore, in spite of the limitation of the model, it is confirmed that the fluctuations of the number of uninterrupted sequences of like elements offer one possible explanation of the data available for PLGA 2.

We may, however, note a similarity between the results for PLGA 2 and those obtained by Eggers and Funck for a molecular weight between that of PLGA 1 and PLGA 2, i.e., in both series of experiments a shift of the maximum of ultrasonic absorption towards high values of the helicity is exhibited. On the other hand, at equal weight concentration, there are less entanglements for the lower molecular weight. Thus, the PLGA 2 solutions are effectively more dilute than those of PLGA 1. Therefore, it may be that the shift of the ultrasonic peak for PLGA 2 and the concomitant appearance of a second relaxation are the result of intramolecular interactions, as discussed earlier in this section in connection with the results of Eggers and Funck.

We intend to carry out further measurements under conditions in which the processes are slower ( $k_{\mathrm{D}}$ smaller). In this way, we hope to be able to study the second relaxation more effectively and evaluate its contribution to the ultrasonic absorption of PLGA with greater accuracy. 


\section{References}

[1] Schwarz, G., Ber. Bunsenges. Phys. Chem. 68 (1964) 843.

[2] CERF, R., J. Physique Colloq. 33 (1972) C 6-99.

[3] CERF, R., Cooperative conformational kinetics of synthetic and biological chain molecules, in Adv. Chem. Phys. ed. Pri-gogine et Rice (Interscience, New York) 1975, vol. XXXIII, p. 73.

[4] Schwarz, G., Ber. Bunsenges. Phys. Chem. 75 (1971) 40.

[5] Schwarz, G., J. Theoret. Biol. 36 (1972) 569.

[6] CERF, R., Dormoy, Y., Michels, B., 15th International Conference on Ultrasound, Prague, July 1976.

[7] Zimm, B. H. and Bragg, J. K., J. Chem. Phys. 31 (1959) 526.
[8] Schwarz, G. and Engel, J., Angew. Chem. 84 (1972) 615.

[9] Michels, B. and Cerf, R., C. R. Hebd. Séan. Acad. Sci. D 274 (1972) 1096.

[10] Michels, B., Thesis, Université Louis-Pasteur, Strasbourg (1972).

[11] Barksdale, A. B. and Stuehr, J. E., J. Am. Chem. Soc. 94 (1972) 3334.

[12] EgGERs, F. and Funck, Th., Studia Biophysica, to appear.

[13] De Gennes, P. G., J. Statist. Phys. 12 (1975) 463.

[14] Rifkind, J. and ApPlequist, J., J. Phys. Chem: 86 (1964) 4207.

[15] NoGUCHI, H. and JEN TSI YANG, Biopolymers 1 (1963) 379. 